\title{
The Symptoms of Herbicidal Action: The Case of Aclonifen
}

\author{
Özgür Kılınç*
}

Department of Plant Production and Technologies, Faculty of Agricultural Sciences and Technologies, Niğde University, 51240 Niğde, Turkey

\section{A R T I C L E IN F O}

\section{Article history:}

Received 20 March 2015

Accepted 01 April 2015

Available online, ISSN: 2148-127X

\section{Keywords.}

Aclonifen

Selectivity

Herbicide

Treatment

Carotenoids

\begin{tabular}{l}
${ }^{*}$ Corresponding Author: \\
\hline E-mail: ozgur.kilinc@ @ nigde.edu.tr
\end{tabular}

\begin{abstract}
A B S T R A C T
The symptoms which were directly bound to the biochemical mode of action of an herbicide, other symptoms result from an indirect consequence of this action. The symptoms of herbicidal action deeply differed and that the climatic factors during the two first weeks after treatment could change definitely the result of the selective herbicide action. The repetitive observation of symptoms allows to inform the farmer about the tolerance or resistance of certain plant species, including the culture, for instance through the appearance of symptoms on the first leaves of the seedlings and their absence in the following leaves. As a whole, the accurate observation of herbicidal symptoms on plants is the essential, rapid and non-expensive analysis of treatment effectiveness at the field scale. The purpose of the current report is to describe the symptoms of a very complex herbicidal action, that of aclonifen involving two modes of action for the same molecule, approximately at the same concentration, and to compare these symptoms under field conditions and under controlled conditions, for a better understanding.
\end{abstract}

\section{Introduction}

Selectively killed weed close to cultivated plants which do have to remain unaffected, is the necessary condition for using herbicides in agronomy (Tissut et al., 2006)

This challenge is generally not a simple one and farmers do have to pay much attention to the effectiveness and selectivity of such a chemical operation which depends on several factors, especially climatic (temperature, rain, hygrometry) and agronomic factors (soil nature, development stage of the culture, weed species and nature of the planted cultivars). That is why the careful observation of the symptoms of the herbicidal action on the culture and on the weeds is a precious source of information (Scalla, 1991; Gauvrit, 1996)

Before the sixties, two main types of symptoms of herbicidal actions were commonly observed which were due to either membrane disruption $\left(\mathrm{H}_{2} \mathrm{SO}_{4}\right.$, dinitrophenols) or synthetic phytohormones (2, 4-D, 2, 4, 5-T, etc.). Membrane disruption obtained through the structural change in the polar fatty acids induced rapid water loss, oxidations and cell death on the places where droplets of the herbicide solution are in close contact with cells of the aerial parts. The major symptom is that of dark spots suggesting a burning effect. (Fedtke, 1982; Bourdin, 1983; Cabanette, 1986; Hascoet and Bourdin, 1988).

The main symptom of phytohormones action was the disorganization of the stem elongation process which affected selectively dicotyledonous plants. This symptom was founded on the structural analogy of the concerned herbicidal family with indol-3-acetic acid (I.A.A.) and on the fact that these herbicides were chemically and biologically stable in marked contrast with I.A.A. (Grossmann, 2000; PerrotRechenmann et al., 2005.)

The observation of such symptoms by the farmers teached them that the herbicidal action may be rapid or slow, depending on the type of biochemical mode of action, that the effectiveness of a herbicide is tightly bound to the development stage of the target-plant, that, in the two main strategies of treatment (pre-emergence and post-emergence), the symptoms of herbicidal action deeply differed and that the climatic factors during the two first weeks after treatment could change definitely the result of the selective herbicide action.

Beside the symptoms which were directly bound to the biochemical mode of action of an herbicide, other symptoms result from an indirect consequence of this action. For instance, the inhibition of cell division often induces an increase in leaf chlorophyll concentration, probably due to a higher nitrogen disponibility (Devine et al., 1993; Tissut et al., 2006). In the same way, the inhibition of protein synthesis may induce a strong anthocyanin synthesis or the accumulation of the secondary pigments, as it is the case for glyposate (Franz et al., 1997)

All these symptoms contribute to evaluate in the field the effectiveness of a herbicide strategy and, often, to deduce the type of herbicide which was used. 
Furthermore, the repetitive observation of symptoms allows to inform the farmer about the tolerance or resistance of certain plant species, including the culture, for instance through the appearance of symptoms on the first leaves of the seedlings and their absence in the following leaves.

As a whole, the accurate observation of herbicidal symptoms on plants is the essential, rapid and nonexpensive analysis of treatment effectiveness at the field scale.

The purpose of the current report is to describe the symptoms of a very complex herbicidal action, that of aclonifen involving two modes of action for the same molecule, approximately at the same concentration, and to compare these symptoms under field conditions and under controlled conditions, for a better understanding.

Aclonifen (2-chloro-6-nitro-3-phenoxyaniline) is an herbicide having the typical diphenylether structure (Figure 1). Among this chemical family numerous derivatives are known herbicides inhibiting one enzyme protoporphyrinogen oxidase (EC 1.3.3.4) in the biochemical pathway leading to chlorophyll or cytochromes biosynthesis (Matringe et al., 1989, Witkowski and Halling, 1989; Arnould and Camadro, 1998).

Several of these diphenylether derivatives were authorized for agronomic uses (acifluorfen, lactofen, oxyfluorfen, etc.) in different countries. One of them, nitrofen has been forbidden since the end of the XXth century (Tomlin, 2006; Tissut et al, 2006)

As shown in Figure 1, aclonifen has a specific substitution allowing a good inhibitory activity on the chlorophyll synthesis pathway but, at the same concentration, it has also a noticeable inhibitory activity on carotenoid biosynthesis. These two modes of action determine a target space inside the plants (Figure 2) which is limited to the aerial parts (Kilinc et al, 2009). Furthermore, these two modes of action seem complementary or even synergistic as they contribute together to induce membrane disruption through;

- The emission of toxic ${ }^{1} \mathrm{O}_{2}$ resulting from plastidial protoporphyrinogen oxidase inhibition (Matrige et al., 1989; Dayan et al., 2001).

- The suppression of the carotenoid plastidial accumulation playing the role of ROS scavengers for the biological membranes (Böger and Sandmann, 1992; Edge et al., 1997; Kilinc et al., 2011).

Such a double complementary mode of action expressed with its highest effectiveness in young plants leads to choose the pre-emergence treatment as the best agronomic strategy. This requires to treatment of the superficial soil layer just after planting the seeds of the culture. Afterwards, aclonifen has to be absorbed by the underground parts and to be transferred towards the forming aerial parts which represent the target space. Light (especially UV light with a $340 \mathrm{~nm} \lambda \max$ ) is required to obtain protoporphyrin IX excitation and formation of ${ }^{1} \mathrm{O}_{2}$. (Duke et al., 1991; Becerril and Duke, 1989; Hess, 2000; Kilinc, 2011).

\section{Materials and Methods}

Plant culture: Six plant species including Zea mays L. var Furio, Triticum aestivum L., Alopecurus myosuroides Hudson, Cucumis sativus L., Sinapis arvensis L. and Vicia faba L. were concurrently cultivated. The weed seeds were supplied by Arbiotech, 35590 Saint Gilles, France. The pre-emergent treatments were comparatively carried out either with pure aclonifen dispersed in water + $2 \%$ dimethylsulfoxide or with the formulated preparation Challenge 600 dispersed in water $\left(30 \mathrm{ml} . \mathrm{m}^{-2}\right)$.

Plants (Alopecurus myosuroides and Brassica napus) were grown in a greenhouse with an electric heating supply when temperature was lower than $10^{\circ} \mathrm{C}$. A 150 $\mu \mathrm{E} . \mathrm{m}^{-2} . \mathrm{s}^{-1}$.PAR light supply was added for 16 hours/day. In other experiments, plants (Zea mays, Cucumis sativus L. and Triticum aestivum) were grown in culture chamber (day: $16 \mathrm{~h}, 25^{\circ} \mathrm{C}$; night: $8 \mathrm{~h}, 18^{\circ} \mathrm{C}$ R.H. $80 \%$ ) and Vicia faba and Sinapis arvensis were cultivated in the fields.

The formulated preparation Challenge 600 (with surfactant and other formulating agents) was used as a preplant treatment at different doses, among which $2.7 \mathrm{~kg}$ a.i/ha in 3001 water which is the conventional concentration for agronomic uses in France.

${ }^{14} \mathrm{C}$ labelling: ${ }^{14} \mathrm{C}$ aclonifen $\left(1167 \mathrm{~Bq} \cdot \mathrm{nmol}^{-1}\right)$ was a generous gift from Bayer CropScience AG Wuppertal Germany. Unlabelled aclonifen was extracted from the commercial product "Challenge 600" (Bayer CropScience) containing $600 \mathrm{~g} / \mathrm{l}$ of a.i. The acetonic extract was fractionated with petrol ether $40-60^{\circ} \mathrm{C}$. The latter solution was evaporated to dryness, dissolved in the minimal amount of acetone and stored at $4{ }^{\circ} \mathrm{C}$ until crystallization. This process was repeated three times and gave pure aclonifen as controlled by TLC chromatography and spectrophotometry.

Critical concentration measurement: Plants were treated by ${ }^{14} \mathrm{C}$ aclonifen at different concentration. Plant fresh samples (aerial and underground parts separately) were submitted to three successive extractions with acetone. The acetonic solutions were evaporated to dryness and redissolved in $70 \% \mathrm{EtOH}$. An aliquot of the dry residual pellet was dissolved with $10 \mathrm{~mL}$ of mineralization mixture, $\mathrm{H}_{2} \mathrm{O}_{2} /$ perchloric acid/ $\mathrm{H}_{2} \mathrm{O}(1 / 1 / 1$, $\mathrm{v} / \mathrm{v} / \mathrm{v}$ ), in closed polyethylene flasks. After 2-3 days of mineralization at $25^{\circ} \mathrm{C}$, the radioactivity of the mineralization mixture as well as that of ethanol $70 \%$ solutions were measured through scintillation counting (1414 Winspectral EG\&G Wallac), using PerkinElmer ULTIMA GOLD AB liquid. During the mineralization step, the absence of ${ }^{14} \mathrm{CO}_{2}$ leaching from the flasks was controlled using a $\mathrm{KOH}$ trap. The critical concentration was the lower concentration inducing lethal symptom.

Autoradiography: TLC chromatography was carried out on silica gel thin layer plates (Macherey-Nagel TLC plates SIL G-25 $0.25 \mathrm{~mm}$ silica gel). Several solvents with different polarities were used especially petrol ether/dichloroethane/EtOH/acetonitrile (4/4/2/1) in which aclonifen $\mathrm{Rf}$ value was 0.9 , and dichloroethane/EtOH/acetonitrile/ $\mathrm{H}_{2} \mathrm{O} \quad(4 / 4 / 2 / 0.1) \quad$ in which different conjugates had a Rf value between 0 and 0.2 . ${ }^{14} \mathrm{C}$-aclonifen derivatives from harvested plant tissues 
were analyzed using PerkinElmer Phosphorimager CycloneTM. The sensitivity limit after one hour exposure was $2 \mathrm{dpm} / \mathrm{mm}^{2}$.

Symptoms observation: The observation of the plants consisted in biometric characters measurement (aerial parts and roots length and weight, number of leaves). Necrosis observation (aspect and number of necrotic spots on leaves), leaf colour evaluation (green, yellow, white), meristematic activity observation and seedlings death.

Leaf fragments in vitro infiltration medium: Cucumber (Cucumis sativus L.) cotyledons and maize (Zea mays) fragments were obtained from seedlings grown at $25^{\circ} \mathrm{C}$, $80-90 \%$ relative humidity, under $150 \mu \mathrm{E} \mathrm{m} \mathrm{m}^{-2} \mathrm{~s}^{-1}$ PAR illumination with $16 / 8 \mathrm{~h}$ light/dark period. Cotyledons were fully developed 12 days after sowing (at this stage the first leaf was growing). Experiments were carried out with rectangular cotyledon pieces $(10 \times 10 \mathrm{~mm})$, as previously described by Routaboul et al., 1990. Cotyledon and maize fragments in Petri dishes were respectively treated with different aqueous solutions of aclonifen and acifluorfen. Petri dishes were placed in vacuo for 15 minutes to facilitate the herbicide penetration. Since diphenyl ether effect is photodependent, one part of the dish was kept in darkness and the other in the light. The appearance of symptoms was monitored during 48 hours.

Field application: The field bean treated and untreated cultivars were sown in the field during the years 2010. The herbicide, aclonifen (Challenge 600) was used as preemergence treatment according to France recommended dose 4 1.ha-1 in 3001 .

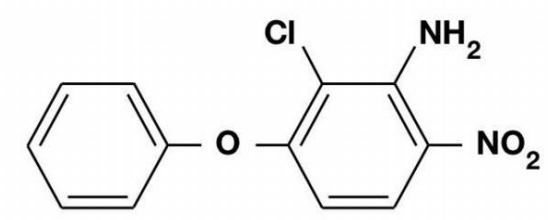

Figure 1 Chemical structure of the studied herbicide aclonifen

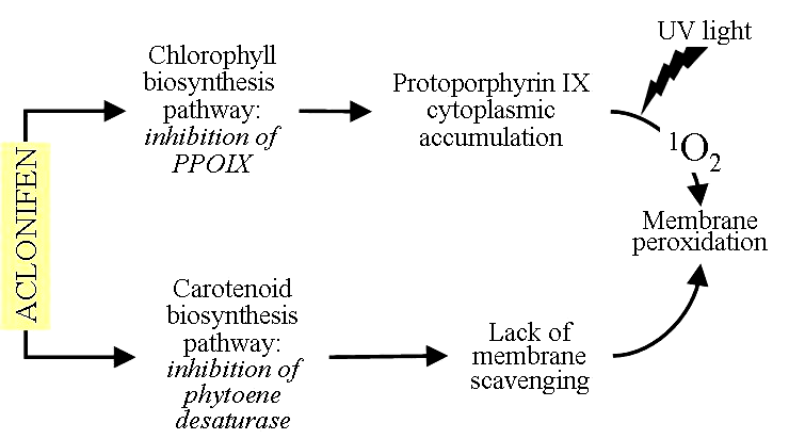

Figure 2 Complementary biochemical effects of aclonifen on plant aerial parts under light.

\section{Results}

Symptoms of aclonifen herbicidal effects in vitro: Experiments in vitro allow to separate the effects of the two modes of action of aclonifen, one being lightdependent $\left({ }^{1} \mathrm{O}_{2}\right.$ production) and the other acting in the dark. Furthermore, with the appropriate surfactants, direct penetration of the a.i. into leaf fragments could be obtained (Routaboul et al., 1990; Ponte-Freitas, 1991), thus avoiding the possible limiting steps controlling root uptake and xylem transfer.

The effects of aclonifen were comparatively studied on cucumber cotyledons fragments and on corn young leaf fragments infiltrated in vacuo with a $10^{-4} \mathrm{M}$ aclonifen solution.

As previously described (Routaboul et al., 1990) the cucumber cotyledon test is a very reliable method demonstrating protoporphyrinogen oxidase inhibition and the corresponding light-dependent necrosis due ${ }^{1} \mathrm{O}_{2}$.

As shown in Figure 3, the necrotic effects of aclonifen $\left(10^{-4} \mathrm{M}\right)$ and acifluorfen $\left(10^{-4} \mathrm{M}\right)$ taken as a typical reference were studied.

In the dark, the two studied a.i. were without effect and the fragments remained alive and green for more than one week. Under light $(250 \mu$ E.PAR, with \% UV 340 $\mathrm{nm})$, the fragments were killed, took a white colour due to oxidation and leaching of the internal components. The same experiment was carried out with corn young leaf fragments with practically, the same results: the light dependent necrosis and bleaching of the fragments was obtained for $10^{-4} \mathrm{M}$ aclonifen or $10^{-4} \mathrm{M}$ acifluorfen.

Symptoms of aclonifen inhibition of carotenoid biosynthesis in wheat seedlings cultivated in the dark: During development of the aerial parts of the seedlings, in the young cells issued from the meristematic activity, proplastids transform into physiologically-active chloroplasts. Inside the thylakoids, the biosynthesis of chlorophyll depends on the presence of light but that of carotenoids has not such a requirement. As a consequence, seedlings developing in the dark form etioplasts accumulating carotenoids but devoid of chlorophyll.

Under such conditions (Figure 4) wheat seedlings treated with aclonifen $\left(10^{-4} \mathrm{M}\right)$ cannot accumulate carotenoids. The news leaves are white and not orange yellow. The appropriate chromatography shows the lack of carotenoids and the accumulation of their biochemical precursor, phytoene.

If acifluorfen replaces aclonifen, only a very low inhibitory effect on carotenoid biosynthesis was observed.

Another interesting point is that the bleached leaves in the dark remain alive for a long time (more than one week), in marked contrast with the leaves exposed to light which were destroyed by ${ }^{1} \mathrm{O}_{2}$.

On the whole, Figure 5 tentatively explains the theoretical scheme of aclonifen herbicidal action. 


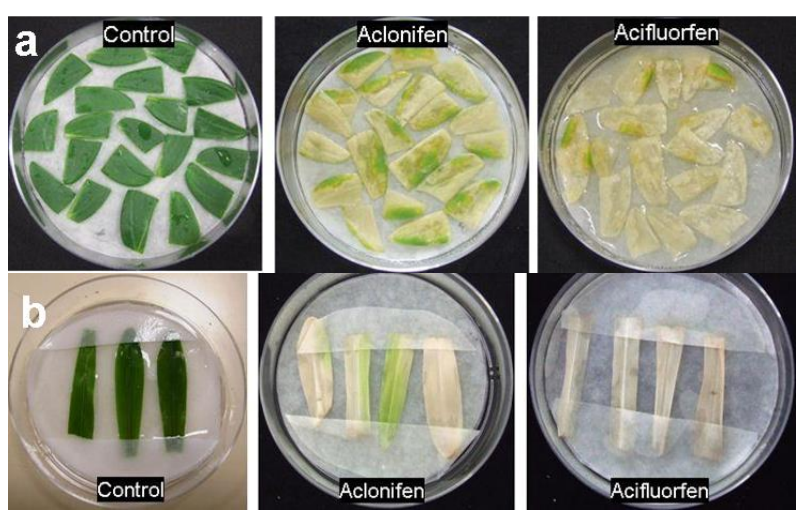

Figure 3 Effect of aclonifen $\left(10^{-4} \mathrm{M}\right)$ and acifluorfen $\left(10^{-4}\right.$ M) on a) cucumber cotyledons and b) corn young leaf fragments cultivated in liquid medium under light at $48 \mathrm{~h}$.
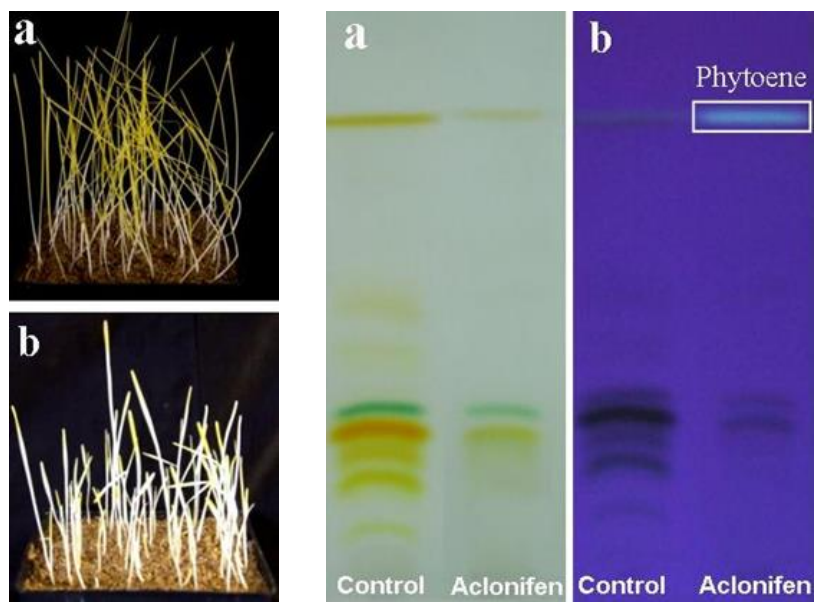

Figure 4: Wheat seedlings grown (a: control, b: aclonifen) in the dark and submitted to a pre-emergence treatment with aclonifen at $2.7 \mathrm{~kg} \cdot \mathrm{ha}^{-1}$. Thin layer chromatography of lipophilic leaf extracts from wheat seedlings grown in the dark. Wheat were treated with $10^{-4} \mathrm{M}$ aclonifen or untreated (control). (a) Carotenoids were visualized under visible light ( $\beta$-carotene: $\operatorname{Rf} 0.94$; lutein: $\operatorname{Rf} 0.37$; zeaxanthin: Rf 0.28; violaxanthin: Rf 0.22). (B). Phytoene accumulation was monitored under UV light $(254 \mathrm{~nm})$. Silica gel plates-solvent: petroleum ether, acetone (10/3: $\mathrm{v} / \mathrm{v})$.

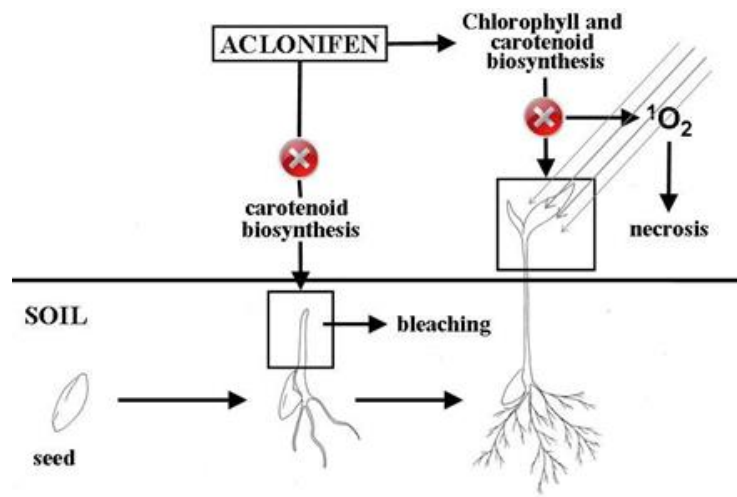

Figure 5 Theoretical scheme explaining the herbicidal mode of action of aclonifen
Symptoms of the herbicidal action of an aclonifen preemergence treatment on seedlings of different species in the greenhouse or in culture chambers:

On cultivated plants or weeds, in the greenhouse or culture chamber, the symptoms of aclonifen agree with Figure 6.

Sinapis arvensis shows the two types of biochemical actions:

- Loss of carotenoid synthesis allowing chlorophyll degradation and bleaching in the cotyledons

- Rapid necroses of the meristematic and elongation zones in the upper part of the young stem (Figure $6 c)$.

It is interesting to note that the two visible symptoms do not affect apparently the same organs. In monocotyledonous species, such as wheat or Alopecurus, another pattern of visible symptoms is obtained which is strongly associated to the growth program of Poaceae plants: The meristematic region, hidden in the centre of the plant, surrounded by previously formed leaves, cannot be observed. Thus, the necrosis effect can be observed only in very young seedlings having absorbed rapidly a sufficient amount of herbicide to reach the critical concentration for membrane disruption. Generally, obtaining the critical concentration requires more time and occurs after the plantlet has formed one or two leaves.

At this stage, a typical symptom of carotenoid biosynthesis inhibition can be seen in the basal part of the leaves which is the part, in Poaceae, where elongation and chlorophyll synthesis occur (Figure 7).

In corn, obtaining bleaching symptoms in leaves $\mathrm{n}^{\circ} 1$ or 2 under experimental conditions is relatively easy. After one week, the newly formed leaves were always normal, showing that the critical concentration could not be reached.

Symptoms of aclonifen action in the field: The symptoms on each plant species observed in the greenhouse can also be seen in the field (Figure 8). However, a new parameter can be rapidly evaluated, which is the global efficiency on the culture. For instance, figure 8 shows comparatively the untreated and treated areas for the weed development and competition between weeds and culture (here wild mustard and field bean) and for the ability of weeds to form a large amount of seeds.

In this case, the efficiency of the herbicide depends evidently on the biochemical efficiency but also on the continuity of the pre-emergence film deposit. This continuity depends heavily on climatic conditions during the first stage of the culture.

\section{Discussion}

Herbicide chemistry and physical properties usually determine how herbicides interact with the biological and physical systems of the plant. Factors determining herbicide efficacy and crop safety are complex and include plant species, plant size, growth stage, soil chemical and physical properties, soil moisture, temperature, and relative humidity (Duke,1990; Devine et al., 1993). 


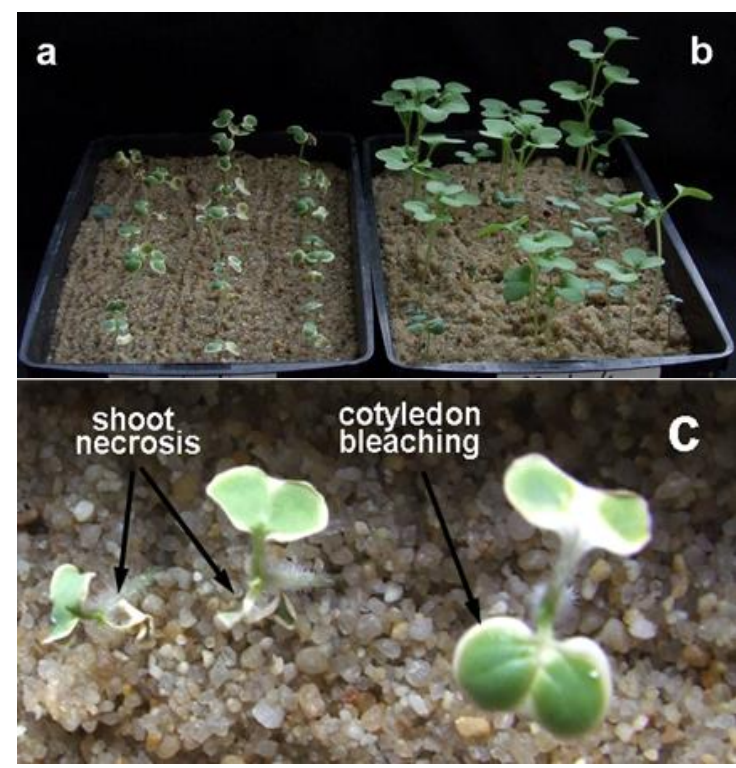

Figure 6 Aclonifen symptoms in Sinapis arvensis seedlings cultivated in culture chamber a: submitted to a pre-emergence treatment with aclonifen at $2.7 \mathrm{~kg} \cdot \mathrm{ha}^{-1} . \mathrm{b}$ : control, c: Symptoms of aclonifen action in Sinapis.arvensis
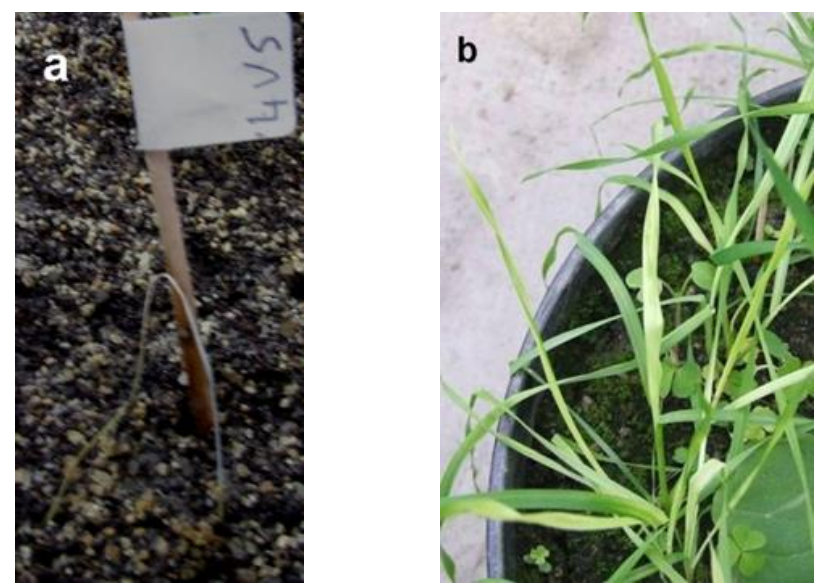

Figure 7 Symptoms of aclonifen action in Alopecurus myosuroides. a) Necrosis of stem tips b) Carotenoid inhibition in the basal parts of the leaves $n^{\circ} 1$ or 2 .

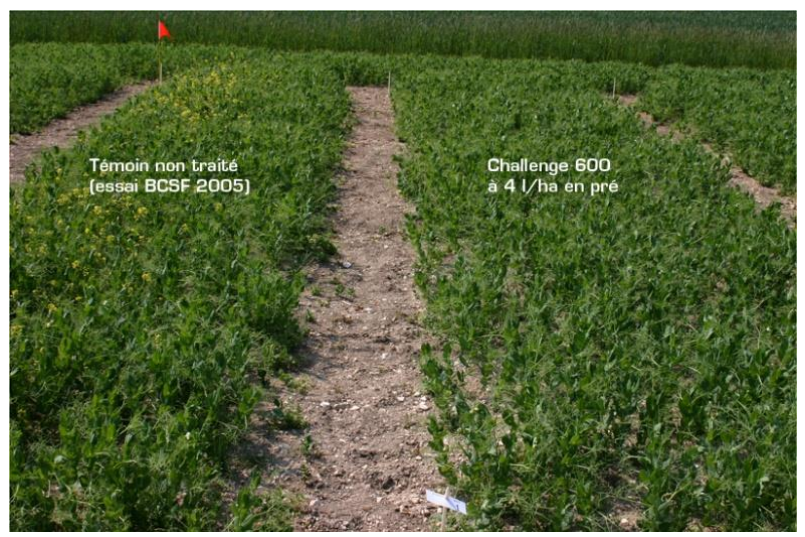

Figure 8 Field bean culture either untreated or treated by aclonifen. $4 \mathrm{~L} / \mathrm{ha}^{-1}$ (wild mustard reaches the flowering stage only in the untreated area)
Herbicide symptoms vary depending on the herbicide, the rate of application, growth stage, type of exposure, and the plant species receptor involved. In general, herbicides with the same mode of action produce similar injury symptoms because the outward appearance of injury is a function of herbicide effect on the plant at the cellular level. Therefore, it is much easier to diagnose symptoms belonging to different herbicide modes of action than herbicides within the same modes of action. In addition, diagnosing herbicide symptoms can be difficult because herbicide symptoms may look very similar to symptoms caused by diseases, nutrient deficiencies, environmental stress, and soil compaction (Fedtke, 1982; Duke, 1990; Böger and Sandmann, 1992).

On pre-emergence application of aclonifen, the susceptible plants starts exhibiting injury symptoms of bleaching, yellowing, or chlorosis between leaf veins, followed by yellowing at the margins; that ultimately leads to tissue death. Older leaves are more affected than newer leaves.

In monocotyledonous species, another pattern of visible symptoms is observed that is strongly associated to the growth program of Poaceae plants: The meristematic region, hidden in the centre of the plant, surrounded by previously formed leaves, cannot be observed. Thus, the necrosis effect can be observed only in very young seedlings having absorbed rapidly a sufficient amount of herbicide to reach the critical concentration for membrane disruption.

The symptoms on each plant species observed in the greenhouse can also be seen in the field. However, a new parameter can be rapidly evaluated, which is the global efficiency on the culture.

Observation of symptoms of the herbicide treatment gives access to fruitful information concerning several points:

- It allows generally to understand what is the mode of biochemical action of the herbicide, as it is the case for aclonifen

- It allows a global evaluation of the sensitivity of the different plant species present in the field and to know the level of culture tolerance for the herbicide.

- It may testify for a tolerance or resistance developing among the weeds flora in field conditions.

- It allows evaluating whether the agronomic practice (i.e. soil mechanical preparation), the climatic conditions and the herbicide formulation were or not optimized.

In the case of aclonifen, an accurate field observation allows even to see the result of the two modes of action. Such observations, which are to be carried out by the farmer himself, seem to be the necessary tool for optimizing the use of herbicides and choosing the lowest efficient doses. 


\section{References}

Arnould S, Camadro JM. 1998. The domain structure of protoporphyrinogen oxidase, the molecular target of diphenyl ether-type herbicides. Proc. Natl. Acad. Sci. U.S.A., 95: 10553 10558.

Becerril J, Duke SO. 1989. Protoporphyrin IX content correlates with activity of photobleaching herbicides. Plant physiol. 90: 1175-1181.

Böger P, Sandmann G. 1992. Target assays for modern herbicides and related phytotoxic compounds. (ed) Lewis Publishers, Florida, USA.

Bourdin J. 1983. Evolution des produits phytosanitaires et des techniques d'application. Phytoma, 353: 6-11.

Cabanettes JP. 1986. Herbicides. Plus d'un demi-siècle d'histoire. La France agricole, 5 sept. 1986, 5-6.

Dayan FE, Romagni JG, Duke SO, Robert IK, William CK. 2001. Protoporphyrinogen Oxidase Inhibitors, Handbook of Pesticide Toxicology (Second Edition). Academic Press, San Diego, pp. 1529.

Devine M, Duke SO, Fedtke C. 1993. Physiology of Herbicide Action. (ed) Prentice Hall, New Jersey, USA.

Duke SO. 1990. Overview of Herbicide Mechanisms of Action. Environmental Health Perspectives 87: 263-271

Duke SO, Lydon J, Becerril JM, Sherman TD, Lehnen LP, Matsumoto H. 1991. Protoporphyrinogen oxidase inhibiting herbicides. Weed Science 39: 345-352.

Edge R, McGarvey DJ, Truscott TG. 1997. The carotenoids as antioxidants - a review. Journal of Photochemistry and Photobiology B: Biology 41: 189-200.

Fedtke C. 1982. In Biochemistry and Physiology of Herbicide Action (C. Fedtke, ed.), Springer, Berlin. pp. 25-33.

Franz JE, Mao MK, Sikorski JA. 1997. Toxicology and environmental properties of glyphosate. In: Glyphosate: A Unique Global Herbicide. American Chemical Society Monographs, Vol. 189. American Chemical Society, Washington, DC, pp. 103-137.
Grossmann K. 2000. Mode of action of auxin herbicides: a new ending to a long, drawn out story. Trends in Plant Science 5: 506-508.

Hascoet M, Bourdin J. 1988. Evolution des produits antiparasitaires et situation actuelle. Phytoma, 400: 68-73.

Hess FD. 2000. Light-dependent herbicides: an overview. Weed Sci. 48: 160-170.

Kilinc Ö, Reynaud S, Perez L, Tissut M, Ravanel P. 2009. Physiological and biochemical modes of action of the diphenylether aclonifen. Pestic Biochem Physiol. 93: 65-71.

Kilinc Ö, Grasset R, Reynaud S. 2011a. The herbicide aclonifen: The complex theoretical bases of sunflower tolerance. Pestic Biochem Physiol. 100: 193-198.

Kilinc Ö. 2011b. Aclonifen: The identikit of a widely used herbicide. Afr. J. Agric. Res., 6: 2411-2419.

Matringe M, Camadro JM, Labbe P, Scalla R. 1989. Protoporphyrinogen oxidase inhibition by three peroxidizing herbicides: Oxadiazon, LS 82-556 and M\&B 39279. FEBS Letters 245, 35-38.

PerrotRechenmann, C, Napier RM,. Gerald L. 2005. Auxins, p. 203 Vitamins \& Hormones, Vol. Volume 72. Academic Press.

Ponte-Freitas A, Haddad G, Tissut M, Ravanel P. 1991. Distribution of Isoproturon a Photosystem II Inhibitor inside Wheat Leaf Fragments. Plant Physiol and Biochem 29: 67-74.

Routaboul JM, Nurit F, Ravanel P, Tissut M. 1990 Effects of bifenox and oxadiazon on isolated chloroplasts, plant mitochondria and leaf pieces. Pestic Sci., 30: 149-158.

Scalla R. 1991. Les herbicides: Mode d'action et principes d'utilisation. INRA, Paris, France, pp, 450.

Tissut M, Delval JM, Ravanel P. 2006. Plantes, herbicides et désherbage ACTA, Paris, France, pp, 635.

Tomlin CDS. 2006. The pesticide manual. Fourteenth Edition. Published by BCPC (British Crop Production Council). Hampshire, UK.

Witkowski DA, Halling BP. 1989. Inhibition of plant protoporphyrinogen oxidase by the herbicide acifluorfenmethyl. Plant Physiol 90: 1239-1242. 